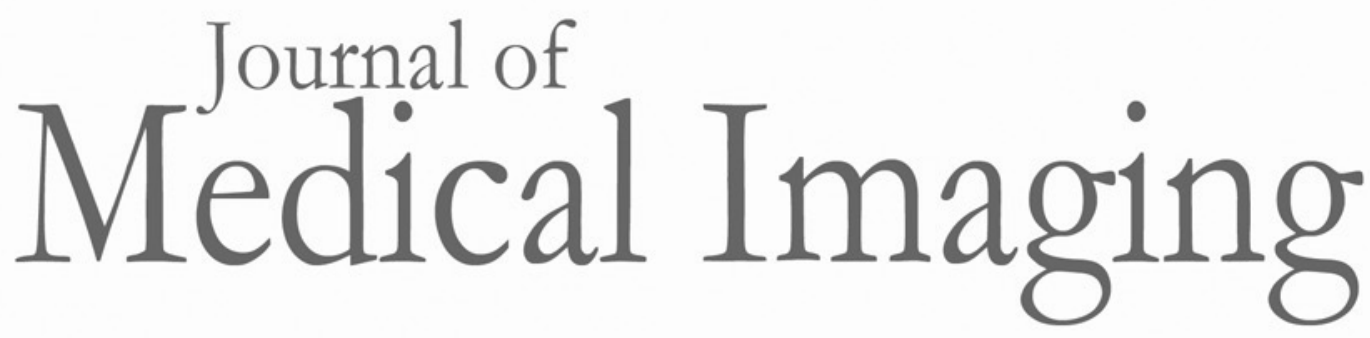

\title{
Quantitative analysis of hypertrophic myocardium using diffusion tensor magnetic resonance imaging
}

Nicholas Tran

Archontis Giannakidis

Grant T. Gullberg

Youngho Seo 


\title{
Quantitative analysis of hypertrophic myocardium using diffusion tensor magnetic resonance imaging
}

\author{
Nicholas Tran, ${ }^{a}$ Archontis Giannakidis, ${ }^{\mathrm{b}, \mathrm{c}, \mathrm{d}}$ Grant T. Gullberg, ${ }^{\mathrm{a}, \mathrm{d}, \mathrm{e}, \star}$ and Youngho Seo ${ }^{\mathrm{a}, \mathrm{d}, \mathrm{e}, \mathrm{f}}$ \\ aUniversity of California, Department of Radiology and Biomedical Imaging, Physics Research Laboratory, 185 Berry Street, Ste 350, \\ San Francisco, United States

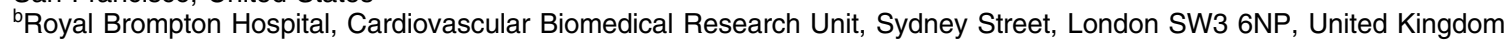 \\ 'National Heart and Lung Institute, Imperial College London, Sydney Street, London SW3 6NP, United Kingdom \\ 'Lawrence Berkeley National Laboratory, Structural Biology and Imaging Department, One Cyclotron Road, Berkeley, California, United States \\ eUniversity of California, Joint Graduate Group in Bioengineering, 1700 4th Street, San Francisco, United States \\ fUniversity of California, Department of Radiation Oncology, 1600 Divisadero Street, San Francisco, United States
}

\begin{abstract}
Systemic hypertension is a causative factor in left ventricular hypertrophy (LVH). This study is motivated by the potential to reverse or manage the dysfunction associated with structural remodeling of the myocardium in this pathology. Using diffusion tensor magnetic resonance imaging, we present an analysis of myocardial fiber and laminar sheet orientation in ex vivo hypertrophic (6 SHR) and normal (5 WKY) rat hearts using the covariance of the diffusion tensor. First, an atlas of normal cardiac microstructure was formed using the WKY b0 images. Then, the SHR and WKY b0 hearts were registered to the atlas. The acquired deformation fields were applied to the SHR and WKY heart tensor fields followed by the preservation of principal direction (PPD) reorientation strategy. A mean tensor field was then formed from the registered WKY tensor images. Calculating the covariance of the registered tensor images about this mean for each heart, the hypertrophic myocardium exhibited significantly increased myocardial fiber derangement $(p=0.017)$ with a mean dispersion of $38.7 \mathrm{deg}$, and an increased dispersion of the laminar sheet normal $(p=0.030)$ of 54.8 deg compared with $34.8 \mathrm{deg}$ and $51.8 \mathrm{deg}$, respectively, in the normal hearts. Results demonstrate significantly altered myocardial fiber and laminar sheet structure in rats with hypertensive LVH. $\odot 2016$ Society of Photo-Optical Instrumentation Engineers (SPIE) [DOI: $10.1117 / 1 . J M I .3 .4 .046001]$
\end{abstract}

Keywords: diffusion tensor magnetic resonance imaging; atlas; left ventricular hypertrophy; spontaneous hypertensive rat. Paper 16111R received Jun. 13, 2016; accepted for publication Sep. 28, 2016; published online Nov. 3, 2016.

\section{Introduction}

Pathological left ventricular hypertrophy (LVH) is a myocardial condition that can arise from chronic systemic hypertension and is associated with oxidative stress, ischemia, and heart failure. ${ }^{1-3}$ In previous studies, histology of the hypertrophic myocardium has demonstrated increased derangement of myocardial fibers. ${ }^{4-6}$ Reduced systolic and diastolic function associated with LVH may be a result of this myocardial fiber derangement and associated pathologies, such as myocardial fibrosis. Analysis of the myocardial microstructure and microstructural changes as a result of hypertension-associated structural remodeling may contribute to a better understanding of the biomechanics and electrical behavior of the pathologic myocardium $^{7}$ and help guide treatment of $\mathrm{LVH}$.

Traditionally, assessments of myocardial fiber directionality have been performed histologically using tissue slices. This technique is destructive, mainly two-dimensional, and sensitive to errors in slide preparation. Diffusion tensor magnetic resonance imaging (DTMRI, or simply DTI) is an imaging technique that is sensitive to the anisotropic diffusion of water in tissue. The sensitivity of DTI to tissue anisotropy allows for nondestructive elucidation of myocardial tissue structure. The diffusion tensor, a $3 \times 3$ symmetric matrix at each voxel in a three-dimensional (3-D) volume, can be estimated using six or more diffusion-sensitizing gradient magnetic resonance images and one unweighted b0 image, and represents the diffusivity of water at a voxel. ${ }^{8}$ Microstructural elements of the tissue, such as myocardial fibers, will inhibit the water diffusion in an anisotropic manner. ${ }^{4,8}$ It has been shown in studies ${ }^{7}$ correlating DTI and tissue histology that the primary eigenvector of the diffusion tensor coincides with the myocardial muscle fiber, while the tertiary eigenvector is aligned with the laminar sheet normal. ${ }^{9,10}$ Thus, the directionality of the myocardial fibers and laminar sheets can be established nondestructively using DTI.

Since in vivo cardiac DTI is difficult due to the sensitivity of diffusion imaging to cardiac motion and flow artifacts, a model of the hypertrophic heart is required for ex vivo imaging. Ex vivo imaging of the healthy human heart is difficult due to the high demand for viable human hearts for transplantation, presenting the problem of obtaining an experimental control. The spontaneously hypertensive rat (SHR) serves as a model for systemic hypertension. ${ }^{11}$ These rats invariably develop LVH in response to chronic hypertension. ${ }^{11}$ We present a voxel-wise comparative analysis of the myocardium in these rats to their normotensive controls, Wistar Kyoto (WKY) rats. ${ }^{6}$ This atlas-based approach, which has been employed previously in cardiac MRI, ${ }^{12,13}$ avoids the reproducibility issues involved with a comparative analysis using parameters derived from manually drawn regions of interest (ROI). 
A recent in vivo DTI study of asymmetric hypertrophic cardiomyopathy in human hearts demonstrated that myocardial fibers retained a generally systolic orientation in both systole and diastole. ${ }^{14}$ The SNR limitations of in vivo imaging are demonstrated by the $8 \mathrm{~mm}$ out-of-plane resolution employed in this study. Our analysis does not seek to quantify these cardiac cycle-dependent changes in fiber orientation; absolute changes in fiber arrangement represent a separate facet of pathophysiology in global hypertensive hypertrophy. ${ }^{6}$ Indeed, analysis of hypertrophic myocardium in rats has demonstrated increased disorder of myocardial laminar sheets and remodeling of the extracellular matrix. ${ }^{15}$

\section{Materials and Methods}

All in vivo animal procedures prior to ex vivo imaging in this study were approved by the Institutional Animal Care and Use Committees (IACUC) of both UCSF and Lawrence Berkeley National Laboratory.

\subsection{Imaging Protocol}

Diffusion tensor images from the hearts of five WKY rats and six SHR rats were collected with most approximately 2 years of age, from 2009 to 2010. These animals were obtained from an unrelated longitudinal study of perfusion, and fatty acid and glucose metabolism dysfunction using PET/CT. ${ }^{16}$ The hearts of all SHRs exhibited LVH, confirmed by in vivo myocardial positron emission tomography studies. ${ }^{16}$ In a second 2-year study from 2011 to 2012, it was again confirmed that the SHR model exhibits abnormal perfusion and metabolism. ${ }^{17}$

Anesthetic overdose of isoflurane was used after which the heart was removed and the chambers flushed with saline solution delivered through the arterial valves. This was directly followed by fixation and storage in formaldehyde solution prior to the ex vivo imaging. The mean time between imaging and fixation was $20 \pm 16$ days for the WKY rats (range 6 to 47 days) and $33 \pm 19$ days for the SHR rats (range 10 to 65 days). The formalin fixation process was performed immediately after the death of the study animals. The time from death to the start of the fixation was less than $1 \mathrm{~min}$. Previous studies have noted that cardiac DTI parameters remain stable for a period of up to 3 days between death and formalin fixation. ${ }^{18,19}$ The ratios of heart/body masses were also obtained in order to confirm the presence of LVH.

Diffusion tensor imaging was performed on a Bruker BioSpec 7T small-animal scanner at the University of Utah for 17 hours using a 12 gradient orientation 3-D spin-echo sequence with a TR/TE of $500 / 19.224 \mathrm{~ms}$, and a nominal $b$-value of $1000 \mathrm{~s} / \mathrm{mm}^{2}$ to achieve $60 \%$ signal attenuation for each data set. Gradient separation was $10 \mathrm{~ms}$, gradient duration was $4 \mathrm{~ms}$, and the maximum strength of the gradient pulse was $30 \mathrm{G} / \mathrm{cm}$ with a slew rate of $1.2 \times 10^{5} \mathrm{G} / \mathrm{cm} / \mathrm{s}$. Image matrix size varied from $160 \times 96 \times 96$ to $169 \times 97 \times 97$ with constant voxel dimensions of $0.156 \times 0.156 \times 0.156 \mathrm{~mm}^{3}$. Following data acquisition, diffusion tensor estimation was performed on the resultant diffusion-weighted imaging data sets using the nonlinear least-squares fitting algorithm. ${ }^{20}$

\subsection{Image Registration}

In order to compare the microstructural differences voxel-byvoxel between the hypertrophic and control hearts, a standard normotensive heart atlas was created as a reference. We chose to construct an atlas using group-wise registration of the b0 anatomical WKY heart image data, to which every b0 and tensor data set from both SHR and WKY hearts would be registered. First, the b0 (unweighted) anatomical image and diffusion tensor data sets for each heart were zero-padded to an image matrix of $169 \times 97 \times 97$ and isotropic voxel dimensions of $0.156 \times 0.156 \times 0.156 \mathrm{~mm}^{3}$ using MATLAB. The b0 images were then masked using a threshold method with an erosion operator applied to remove endocardial and epicardial signal.

For each WKY heart, the b0 anatomical MRI image data were converted to an 8-bit grayscale image in the analyze format using a custom MATLAB implementation. Then a landmarkbased rigid registration was performed in the DiffeoMap software package $^{21}$ with the apex of the heart and papillary muscles used as landmarks. The resultant data were used as an input to GLIRT $^{22}$ (software package from University of North CarolinaChapel Hill), which used an iterative diffeomorphic demons transformation algorithm ${ }^{23}$ to produce a group mean image.

GLIRT first computed a working group average from the rigidly aligned anatomical WKY hearts. This working average was used as the template to compute the first diffeomorphic transform of the hearts. A second working average was then taken of the transformed hearts, and was used as the template image for a second diffeomorphic transform (see Fig. 1). This process was iterated until the group average converged, which required 10 iterations. The Jaccard similarity ${ }^{24}$ between the thresholded 9th and 10th iterations was calculated to be 1 , and convergence verified by visual inspection.

The resulting final group mean of anatomical WKY b0 images was then used as the reference image to which the anatomical SHR and WKY heart b0 images were coregistered. Automatic registration was performed using the DiffeoMap and DTIstudio software packages from Johns Hopkins University. ${ }^{21}$ After manual histogram intensity matching was performed on each anatomical image, the automatic large deformation diffeomorphic metric mapping (LDDMM) function of DiffeoMap was used to compute the final registration. ${ }^{21}$ This registration process produced deformation fields that were used to transform the diffusion tensor data of each SHR and WKY heart by adding the deformations to the unregistered but aligned tensor images. Then, a tensor reorientation was performed using the preservation of principal direction (PPD) strategy. ${ }^{25}$ The tensor field of the myocardium was segmented using a global threshold. The registered diffusion tensor data for the group of normal WKY rat hearts were used to compute, as described in the next section, an average tensor atlas.

The quality of the overall registration was evaluated using the Jaccard similarity coefficient ${ }^{24}$ calculated by dividing the size (number of voxels) of the intersection of two sample sets by the size of their union. (The coefficient varies between 0 for two sets that do not intersect at all and 1 for sets that are identical.) This was obtained for each sampled heart using MATLAB. The registered tensor field was compared with the group mean (average tensor atlas) to assess the quality of registration. ${ }^{26}$ The average Jaccard similarity coefficient was 0.71 for WKY hearts and 0.69 for SHR hearts. No statistically significant difference in Jaccard similarity coefficients was observed.

Since the registered tensor data now shared spatial and anatomical coordinates, a voxel-wise comparison could be drawn between the hypertrophic rat hearts (SHR hearts) and the normotensive WKY hearts. 


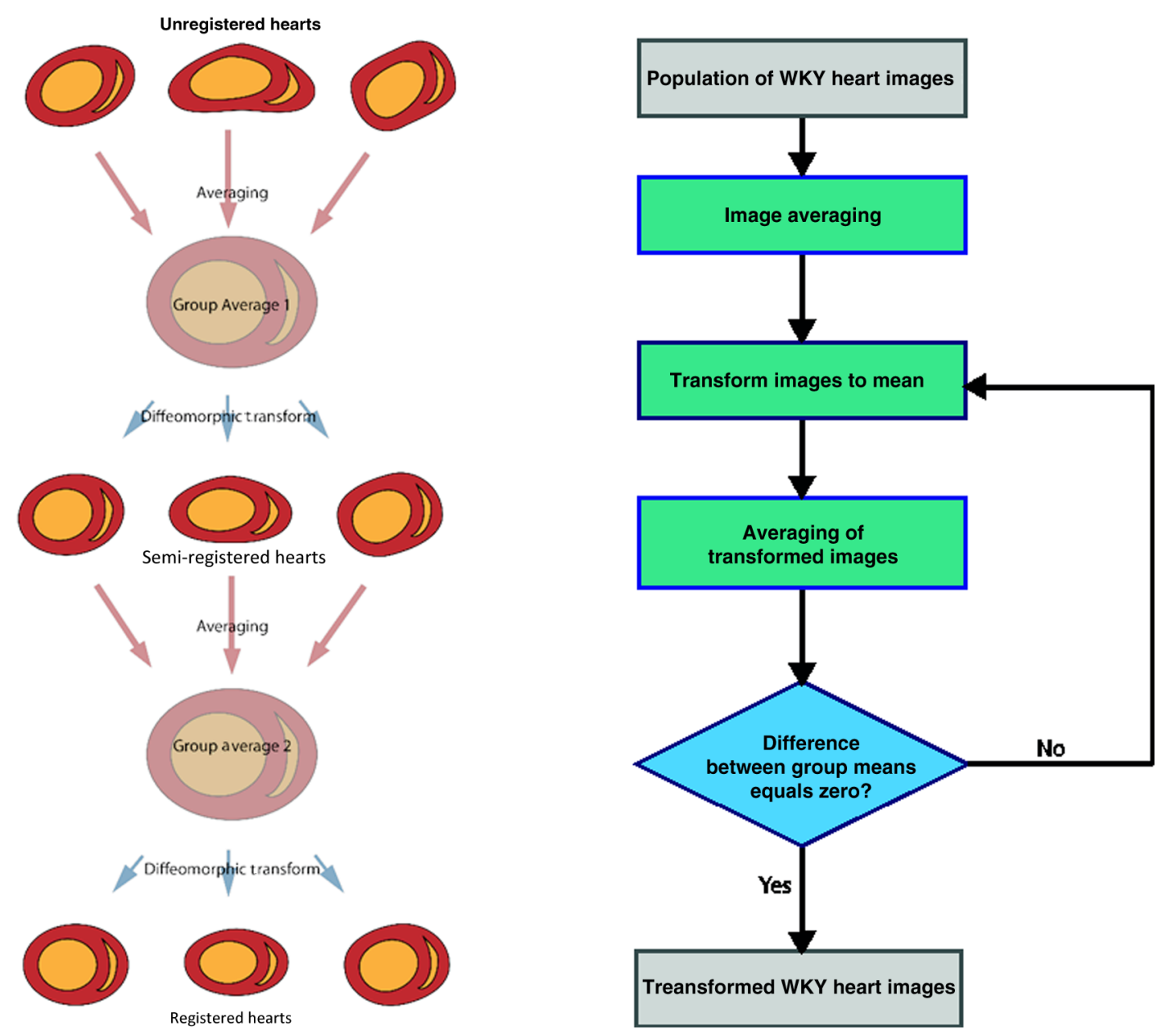

Fig. 1 Iterative diffeomorphic registration performed by GLIRT. Workflow of the process used to produce the deformation fields is shown on the left as a schematic and on the right as a block diagram. The initial unregistered hearts are the hearts aligned using the landmarked rigid registration.

\subsection{Log-Euclidean Tensor Averaging}

Following registration of the tensor images, an average WKY tensor image was computed for the tensor atlas using the registered WKY tensor image data. Due to the non-Euclidean nature of the diffusion tensor space, Euclidean element-wise averaging of the tensor at each voxel produces inaccurate results, ${ }^{27}$ which can lead to misleadingly inflated diffusivity. Tensor averaging was implemented in MATLAB following the log-Euclidean framework, ${ }^{27}$ which is a computationally efficient system that avoids these problems of tensor swelling and asymmetry.

Since the diffusion tensor is by definition symmetric positive-definite, its matrix logarithm can be computed by diagonalizing the tensor

$D=R^{\mathrm{T}} S R$

where $D$, the diffusion tensor, is decomposed into the rotation matrix $R$ and the matrix $S$ containing the tensor eigenvalues along the diagonal. $S^{\prime}$ is then computed by taking the natural logarithm of each of the eigenvalues

$S_{i i}^{\prime}=\log \left(S_{i i}\right)$

for $i=1,2,3$. The matrix logarithm at a given voxel $x$ is then $\log [D(x)]=R^{\mathrm{T}} S^{\prime} R$

The mean tensor can then be calculated by averaging the tensor logarithms for each voxel $x$ across $N$ tensor images and taking the matrix exponent ${ }^{27}$

$\bar{D}_{\log }(x)=\exp \left\{\frac{1}{N} \sum_{n=1}^{N} \log \left[D_{n}(x)\right]\right\}$.

This computation was performed for each voxel across a $160 \times 96 \times 96$ image matrix using all the WKY tensor data after image registration. The result of this was the WKY heart tensor atlas that served as the basis for the tensor dispersion comparison.

\subsection{Myocardial Dispersion Measures}

The covariance matrix between each tensor and the average tensor was then computed voxel-by-voxel for the same voxels in the atlas volume, again using our custom-written MATLAB implementation. Compared to scalar DTI measures, such as the fractional anisotropy (FA), the covariance method describes the variability in orientation of the tensor about some reference rather than the simple magnitude of the anisotropy. This information allows differences in myocardial fiber directionality to 
be comparatively analyzed, potentially providing greater insight than can be obtained from the orientation-invariant intravoxel information provided by FA. The covariance $\Sigma$ for $n=$ $1, \ldots, N$ tensor images with mean tensor $\bar{D}_{\log }$ is given by the following equations: ${ }^{10}$

$$
\begin{aligned}
& \Delta D_{n}(x)=\log \left[D_{n}(x)\right]-\log \left[\bar{D}_{\log }(x)\right] \\
& \operatorname{vec}(D)=\left[D_{11}, \sqrt{2} D_{21}, D_{22}, \sqrt{2} D_{31}, \sqrt{2} D_{32}, D_{33}\right]^{\mathrm{T}} \\
& \Sigma(x)=\frac{1}{N-1} \sum_{n=1}^{N} \operatorname{vec}\left[\Delta D_{n}(x)\right] \operatorname{vec}\left[\Delta D_{n}(x)\right]^{\mathrm{T}} .
\end{aligned}
$$

At a given voxel, the components of the $6 \times 6$ covariance matrix in Eq. (7), in the directions of the planes normal to the three eigenvectors of the mean tensor, correspond to the dispersions of the three equivalent eigenvectors of the sample tensor $D$. Thus, the orthonormal basis of the mean diffusion tensor is computed [Eqs. (8)-(10)], with the motivation of projecting the covariance matrix onto the appropriate directions to compute the eigenvector variability

$$
\begin{aligned}
& W_{23}=\frac{1}{\sqrt{2}}\left(\nu_{3} \nu_{2}^{\mathrm{T}}+\nu_{2} \nu_{3}^{\mathrm{T}}\right), \\
& W_{13}=\frac{1}{\sqrt{2}}\left(\nu_{3} \nu_{1}^{\mathrm{T}}+\nu_{3} \nu_{1}^{\mathrm{T}}\right), \\
& W_{12}=\frac{1}{\sqrt{2}}\left(\nu_{2} \nu_{1}^{\mathrm{T}}+\nu_{1} \nu_{2}^{\mathrm{T}}\right),
\end{aligned}
$$

where $\nu_{i}, i=1,2,3$ are the three eigenvectors of the mean tensor $\bar{D}_{\log }$.

The three planes defined by sets of these orthonormal vectors $W_{i j}$ correspond to the three planes normal to the eigenvectors of the average tensor. By computing the components of the covariance matrix in these planes, the variability of the tensor eigenvectors about the average eigenvectors can be calculated by ${ }^{10}$

$$
\begin{aligned}
& E\left(\varepsilon_{23}^{2}\right)=\frac{1}{2\left(\lambda_{2}-\lambda_{3}\right)^{2}}\left[\operatorname{vec}\left(W_{23}\right)^{\mathrm{T}} \cdot \Sigma \cdot \operatorname{vec}\left(W_{23}\right)\right], \\
& E\left(\varepsilon_{13}^{2}\right)=\frac{1}{2\left(\lambda_{1}-\lambda_{3}\right)^{2}}\left[\operatorname{vec}\left(W_{13}\right)^{\mathrm{T}} \cdot \Sigma \cdot \operatorname{vec}\left(W_{13}\right)\right], \\
& E\left(\varepsilon_{12}^{2}\right)=\frac{1}{2\left(\lambda_{1}-\lambda_{2}\right)^{2}}\left[\operatorname{vec}\left(W_{12}\right)^{\mathrm{T}} \cdot \Sigma \cdot \operatorname{vec}\left(W_{12}\right)\right],
\end{aligned}
$$

where $\lambda_{i}=\log \left(d_{i}\right)$ and $d_{i}, i=1,2,3$ are the eigenvalues of the mean tensor $\bar{D}_{\log }$.

Equation (11) describes the variation around the mean tertiary eigenvector corresponding to the mean laminar sheet normal. ${ }^{10}$ This provides a measure of the dispersion of the laminar sheet. Equations (12) and (13) describe the variation around the mean secondary and mean tertiary eigenvectors in planes containing the primary eigenvector. This provides measures of the dispersion of the fiber orientation about these average eigenvectors. The variable $\varepsilon_{i j}$ represents the tangent of the angles of the eigenvectors $\nu_{j}$ about the corresponding mean eigenvector $\nu_{i}$. By taking the inverse tangent, the angular variability of the sampled tensor eigenvectors about the mean can be obtained. One can imagine this as a set of cones of uncertainty surrounding each of the mean eigenvectors. This method was implemented to obtain fiber and laminar sheet dispersion measures for each heart in the WKY and SHR populations.

\subsection{Statistical Analysis}

The SNR was measured in b0 images as the quotient of the mean signal intensity of the myocardium divided by the standard deviation of the largest possible ROI placed in the image background. All estimated values were expressed as mean $\pm \mathrm{SD}$. $p$-values were calculated using a Wilcoxon rank-sum test because the sample size was small and we could not assume the data to be normally distributed. Any $p$-value $<0.05$ was considered statistically significant.

\section{Results}

The dispersion of the myocardial fibers was evaluated using Eq. (12) to provide structural information about the variability of the myocardial fiber orientation in the remodeled hypertrophic myocardium. An example of the spatial distribution of this variability is shown in Fig. 2 as a mapping of the angular dispersion.

Visual inspection of these dispersion maps suggests that the hypertrophic hearts have a higher degree of angular dispersion than the normotensive hearts. To confirm this, the mean fiber variability was calculated for each heart. In order to exclude low-signal edge voxels, each data set was first masked using the thresholded anatomical group mean image to which an erosion operator (shown in Fig. 3) was applied.

The mean fiber dispersion was higher in the hypertrophic hearts than in the normotensive hearts, with some intrapopulation variation (Fig. 4). The average fiber dispersion across all (a)

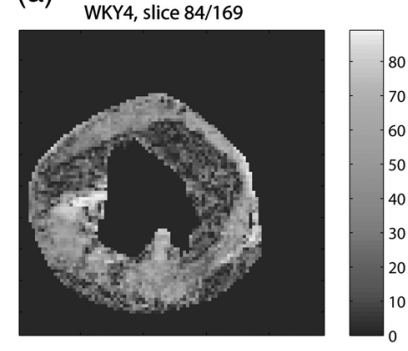

WKY8, slice $84 / 169$

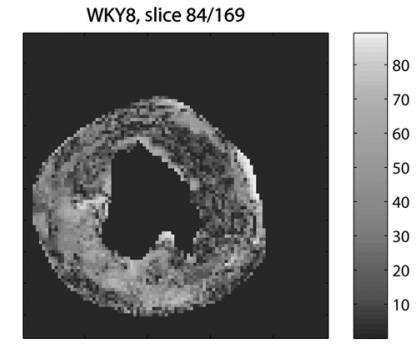

(b)

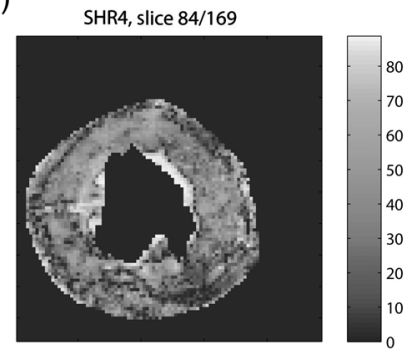

SHR1, slice 84/169

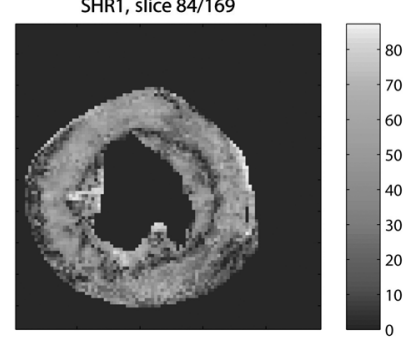

Fig. 2 Masked myocardial fiber dispersion map. Fiber dispersion values mapped for selected axial slice through selected (a) WKY and (b) SHR hearts. Black indicates low dispersion whereas white indicates high dispersion (values in degrees). 
(a) Group mean (intensity image)

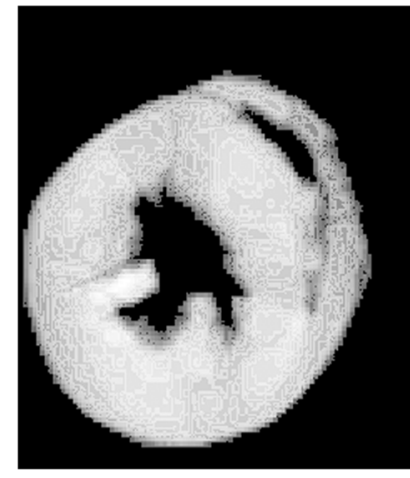

(b) Masked group mean

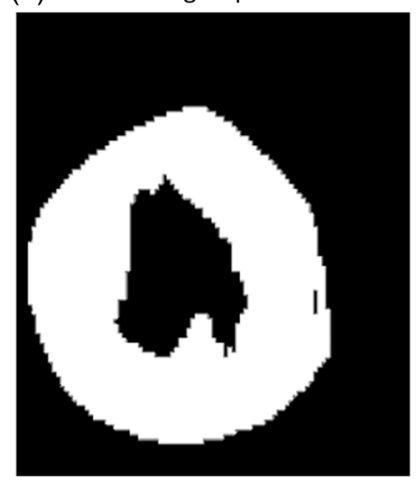

Fig. 3 Group mean images. Grayscale and thresholded group mean image for selected short axis slice.

wild-type hearts was $34.8 \mathrm{deg}$. This was lower than the average fiber dispersion for the hypertrophic hearts, which had an overall mean of dispersion of $38.7 \mathrm{deg}$. The $p$-value obtained using a Wilcoxon rank-sum test was 0.017 . An overall voxel distribution of myocardial fiber angles was also plotted for the normal WKY and hypertrophic hearts in Fig. 5, normalized by dividing each bin count by the total number of voxels for the corresponding population. The histogram shows an increased proportion of high-dispersion voxels in the hypertrophic population compared to the normal type. Global fiber dispersion data for each WKY and SHR sample are presented in Tables 1 and 2, respectively. The mean fiber dispersion was also measured over the 17 AHA ROIs, ${ }^{28}$ defined manually using MATLAB. The average dispersion over each region of interest is reported in Table 3 for both WKY and SHR rats. LVH manifests as a symmetric global hypertrophy ${ }^{29}$ and correspondingly, increased dispersion measures are noted over the majority of the 17 ROIs.

This same data analysis was performed to measure the variability of the laminar sheet [i.e., using the component of the covariance in Eq. (11) of the variation about the third mean diffusion tensor eigenvector]. These measures show increased variability compared to the primary eigenvector (see Fig. 6). As with the fiber orientation dispersion, the comparative difference between WKY and SHR populations is mirrored in the analysis of the laminar sheet angular dispersion, with characteristic increased dispersion in the hypertrophic population shown

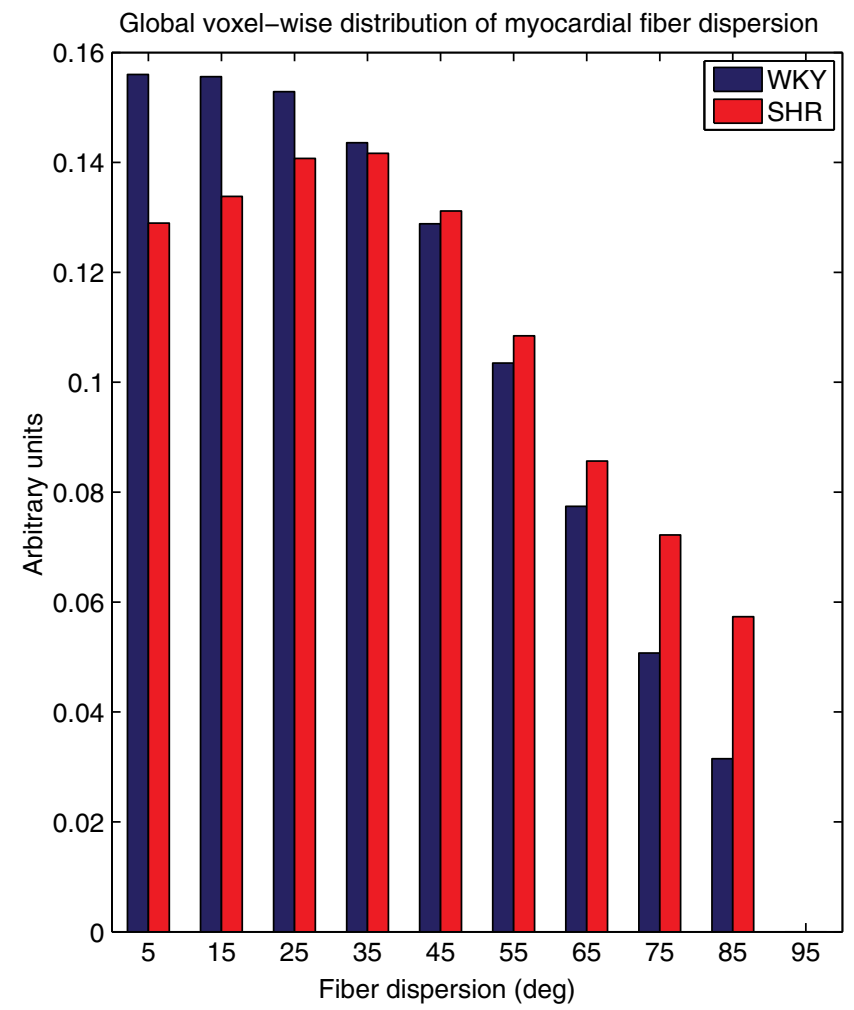

Fig. 5 Histogram of myocardial fiber dispersion angles. Values presented for WKY and SHR hearts, measured in degrees.

in Fig. 7. The calculated dispersion of the laminar sheet normal in the wild-type population was $51.6 \mathrm{deg}$, compared to $54.8 \mathrm{deg}$ in the hypertrophic population. The $p$-value obtained using a Wilcoxon rank-sum test was 0.030. Global laminar sheet dispersion data for each WKY and SHR sample are presented in Tables 4 and 5, respectively.

The ratios of the mass of the excised heart/body mass for the WKY and SHR rat populations are presented in Tables 6 and 7, respectively, with the ages of the rats at time of euthanasia. The mean dispersion is also plotted against heart/body mass ratio in Fig. 8. The WKY samples appear to have lower heart/body mass ratios than the SHR population; however, the $p$-value of 0.067
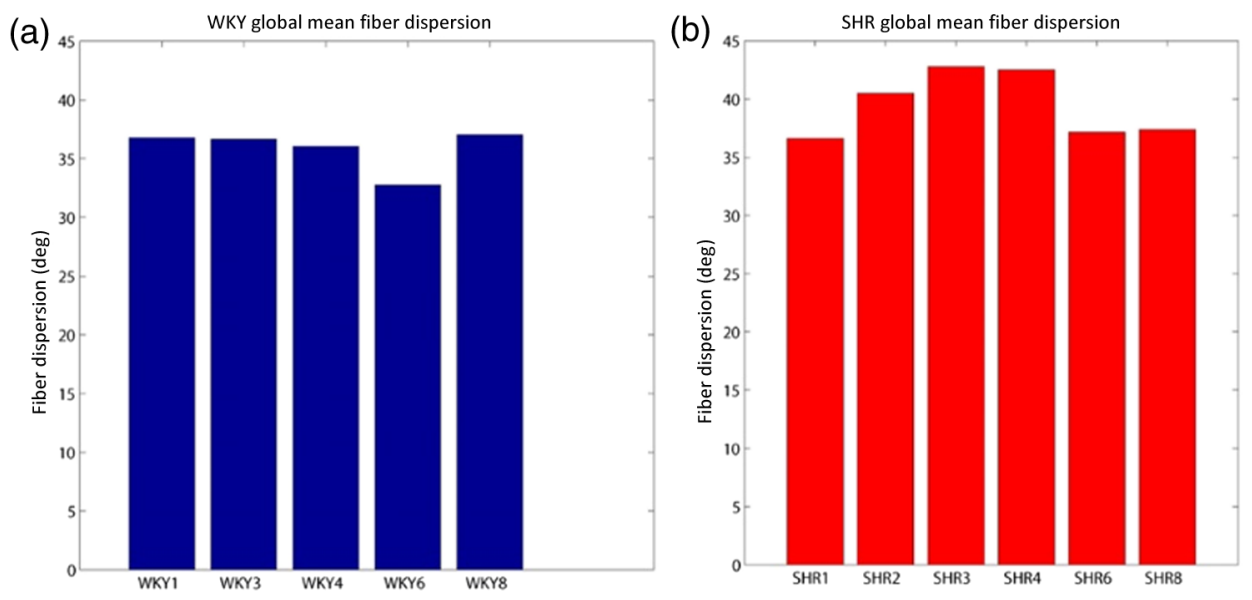

Fig. 4 Mean myocardial fiber dispersion for (a) WKY and (b) SHR hearts. Values measured in degrees. WKY hearts are presented in blue, SHR in red. 
Table 1 Global fiber dispersion measures for WKY rats.

\begin{tabular}{lcc} 
Sample & Global mean dispersion (deg) & Standard deviation \\
\hline WKY1 & 36.0 & 20.66 \\
WKY3 & 35.5 & 22.62 \\
WKY4 & 34.8 & 20.65 \\
WKY6 & 31.8 & 19.41 \\
WKY8 & 36.0 & 22.07 \\
\hline
\end{tabular}

Table 2 Global fiber dispersion measures for SHR rats.

\begin{tabular}{lcc} 
Sample & Global mean dispersion (deg) & Standard deviation \\
\hline SHR1 & 35.8 & 19.73 \\
SHR2 & 39.6 & 22.03 \\
SHR3 & 42.4 & 22.57 \\
SHR4 & 41.8 & 24.68 \\
SHR6 & 36.3 & 21.29 \\
SHR8 & 36.7 & 23.17 \\
\hline
\end{tabular}

Table 3 Regional fiber dispersion measures for WKY and SHR rats.

\begin{tabular}{|c|c|c|}
\hline AHA zone & WKY dispersion (deg) & SHR dispersion (deg) \\
\hline 1 & 36.7 & 41.3 \\
\hline 2 & 29.3 & 31.6 \\
\hline 3 & 29.7 & 34.9 \\
\hline 4 & 37.0 & 38.1 \\
\hline 5 & 36.5 & 38.4 \\
\hline 6 & 35.0 & 42.0 \\
\hline 7 & 37.7 & 38.4 \\
\hline 8 & 31.5 & 33.8 \\
\hline 9 & 35.7 & 37.3 \\
\hline 10 & 33.1 & 32.9 \\
\hline 11 & 37.6 & 40.3 \\
\hline 12 & 30.6 & 34.7 \\
\hline 13 & 27.9 & 30.3 \\
\hline 14 & 29.9 & 30.6 \\
\hline 15 & 29.8 & 34.1 \\
\hline 16 & 28.6 & 35.3 \\
\hline 17 & 25.1 & 38.0 \\
\hline
\end{tabular}

(a)

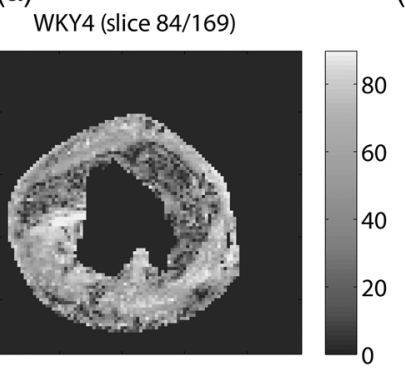

WKY8 (slice 84/169)

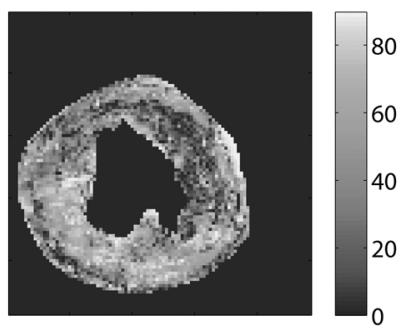

(b)

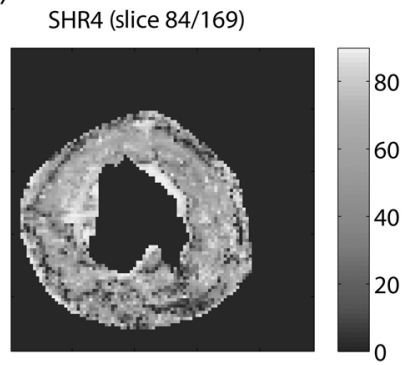

g. 6 Masked laminar sheet dispersion map. Selected axial slice through selected (a) WKY and (b) SHR hearts are presented. Black indicates low dispersion, whereas white indicates high dispersion (values in degrees).

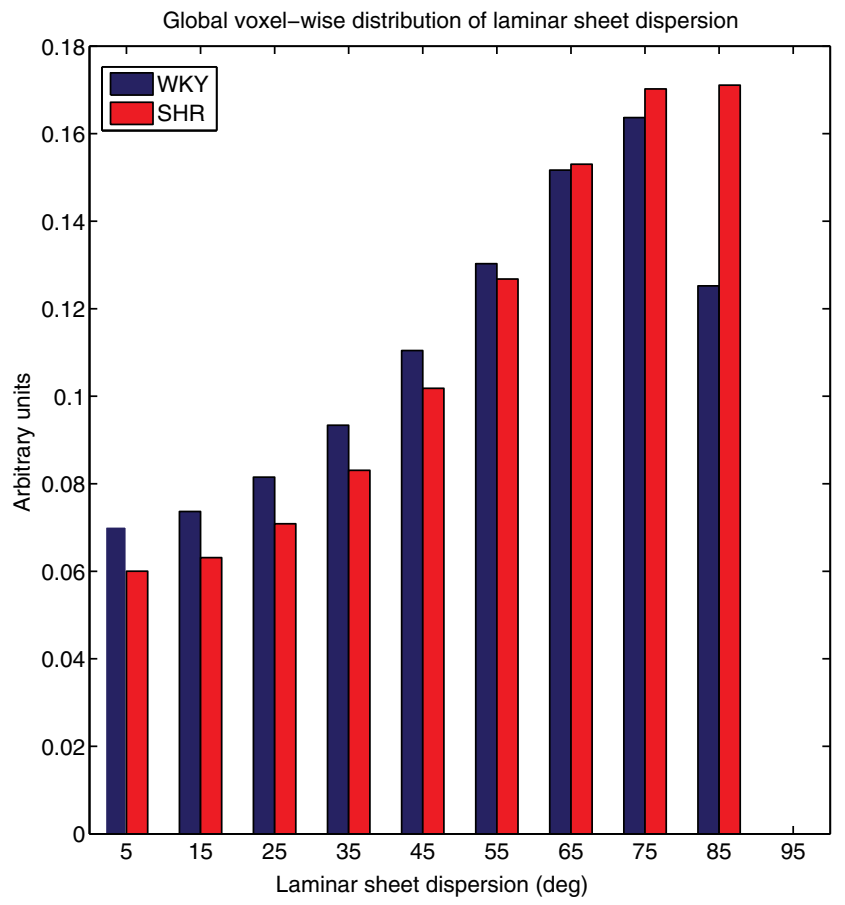

Fig. 7 Histogram of laminar sheet dispersion angles for WKY and SHR hearts. Values measured in degrees.

obtained using the nonparametric test for equal means for heart/ body mass ratio was not significant for this small sample size.

\section{Discussion}

A statistically significant difference was observed in the myocardial fiber and laminar sheet dispersion between the normal wild-type and the hypertrophic population $(p<0.05)$. The dispersion measures were obtained using DT images acquired 
Tran et al.: Quantitative analysis of hypertrophic myocardium using diffusion tensor magnetic resonance imaging

Table 4 Global sheet dispersion measures for WKY rats.

\begin{tabular}{lcc} 
Sample & Global mean dispersion (deg) & Standard deviation \\
\hline WKY1 & 52.5 & 23.89 \\
WKY3 & 52.3 & 24.84 \\
WKY4 & 52.0 & 23.21 \\
WKY6 & 49.2 & 23.59 \\
WKY8 & 52.4 & 24.51 \\
\hline
\end{tabular}

Table 5 Global sheet dispersion measures for SHR rats.

\begin{tabular}{lcc} 
Sample & Global mean dispersion (deg) & Standard deviation \\
\hline SHR1 & 53.5 & 22.71 \\
SHR2 & 54.9 & 24.06 \\
SHR3 & 58.5 & 23.43 \\
SHR4 & 57.4 & 24.34 \\
SHR6 & 52.6 & 24.31 \\
SHR8 & 53.9 & 24.55 \\
\hline
\end{tabular}

Table 6 Ratios of excised heart mass/body mass for WKY rats.

\begin{tabular}{lcc}
\hline & Heart/body mass ratio & Age (months) \\
\hline WKY1 & 0.00335 & 7 \\
WKY3 & 0.00478 & 18 \\
WKY4 & 0.00581 & 7 \\
WKY6 & 0.0031 & 23.5 \\
WKY8 & 0.00342 & 15.5 \\
Average & 0.00409 & 14.3 \\
SD & 0.00104 & 6.42 \\
\hline
\end{tabular}

for $17 \mathrm{~h}$ resulting in excellent image quality and excellent values for the SNR measured in b0 images ranging between 107 and 137 with means of 119 . This lengthy imaging time is not possible for in vivo imaging; however, the approach provides structural information that through computer simulations one can extrapolate information about diastolic and systolic phases of the cardiac cycle. This is the first cardiac DTI paper that uses a cardiac tensor atlas and the covariance matrix to characterize cardiac disease by measuring structural variations of fiber and laminar sheets.

The GLIRT algorithm first produced an atlas from the WKY scalar b0 images. (These images were first aligned using the landmark rigid registration to the apex and papillary muscles.) The atlas was then used to register both WKY and SHR b0 heart images using LDDMM. This provided deformation fields, which were then added to the aligned WKY and the SHR tensor
Table 7 Ratios of excised heart mass/body mass for SHR rats.

\begin{tabular}{lcc} 
Sample & Heart/body mass ratio & Age (months) \\
\hline SHR1 & 0.0044 & 7 \\
SHR2 & 0.00637 & 19.25 \\
SHR3 & 0.00784 & 17.25 \\
SHR4 & 0.00627 & 19 \\
SHR6 & 0.00483 & 16.5 \\
SHR8 & 0.0044 & 15.5 \\
Average & 0.00568 & 15.71 \\
SD & 0.00126 & 4.12 \\
\hline
\end{tabular}

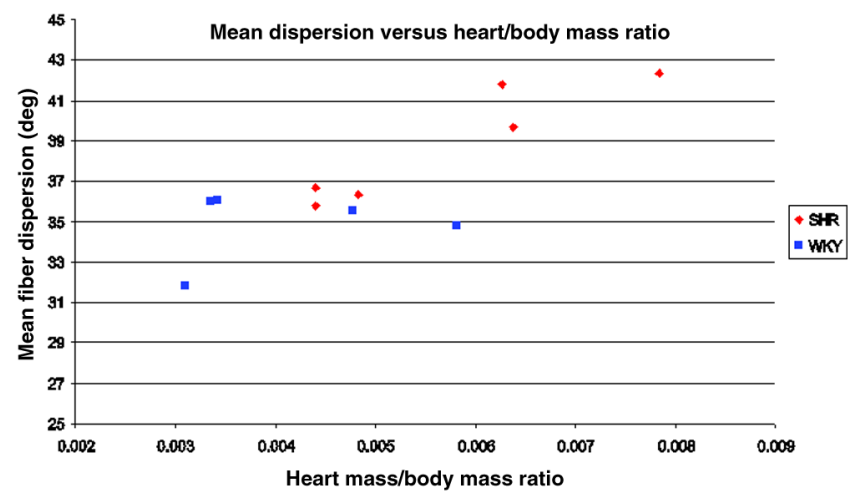

Fig. 8 Mean fiber dispersion of WKY and SHR rats plotted against heart/body mass ratio. The heart/body mass ratio is obtained by dividing the mass of the excised heart by the overall body mass of the rat.

images to provide registered tensor images for each rat type. These tensor images were then followed by the PPD reorientation strategy. ${ }^{10}$ Using the diffusion data from only the WKY hearts, (4) was used to calculate the average tensor. Then, using this average tensor, Eqs. (12) and (11) were applied to the registered WKY tensor images and to the registered SHR tensor images to calculate the fiber and laminar sheet variability for each rat. The registration could have also been performed by using the tensor data to create an atlas for registration..$^{30,31}$ However, we chose to apply to tensor images the deformation fields acquired from the registration of scalar b0 images. ${ }^{10}$ Even though there are other possibilities for tensor registration, this approach has been well tested, and the software ${ }^{21,22}$ is readily available.

The most prevalent morphological characteristic of $\mathrm{LVH}$ caused by arterial hypertension is the symmetric thickening of the LV muscle wall accompanied by an increase in mass. ${ }^{32}$ The addition of sarcomeres also produces an increase in the size of the myocytes and molecular changes to the surrounding collagen network. In the nonmyocyte connective network of the myocardium, these structural changes include an elevated collagen concentration. ${ }^{33}$ This collagen remodeling as a result of hypertensive LVH was observed early on by Weber et al. ${ }^{33}$ and leads to increased cardiac microstructure disorganization. This causes the changes in DTI measures that we observed. Although the exact pathophysiology in these hypertrophic hearts are still an open question, the gross structural remodeling 
in LVH is a change collectively brought about by molecular, cellular, and biochemical events. ${ }^{34,35}$

Traditional scalar measures, such as FA and mean diffusivities (MD), are used to describe in vivo the degree of anisotropy in cardiac disease. This is typically used because these measures do not require the lengthy time necessary for diffusion tensor imaging. Altered measures of FA and MD along with primary (D1), secondary (D2), and tertiary (D3) diffusivities were used to demonstrate a correlation with fibrosis and collagen deposition in patients with idiopathic dilated cardiomyopathy. ${ }^{36}$ Other measures, such as a decrease in circumferential strain, have been used as a marker of LVH associated with increased interstitial fibrosis. ${ }^{37-39} \mathrm{~T} 1$ mapping ${ }^{40-42}$ has also been used to characterize fibrosis in LVH and early gadolinium enhancement ${ }^{43}$ has also been suggested as a potential indicator of myocardial damage in hypertrophic cardiomyopathy.

Tensor measures, however, provide 3-D structure information for developing models to study the electromechanical effects of structural remodeling. The proposed measures in this paper are well established for describing the orientation of the motion of water molecules and, hence, the organization of the fiber and the sheet structure of the myocardium. If fiber-tracking methods are also applied to diffusion tensor data, this can provide an excellent visualization and discrete representation of fiber and sheet structures. ${ }^{44}$ Fusion of the dispersion measures obtained in this paper and fiber-tracking results from the corresponding diffusion tensor data could provide FE computer models for further study into the spatial localization of the pathology and its impact on heart function. These 3-D FE models that incorporate fiber and laminar sheet orientation variability from diffusion tensor data can then be used to study how structural changes reduce myocardial function through computer simulations.

In previous work, we performed computer simulations ${ }^{6}$ of the mechanical effects associated with fiber disarray by making use of our FE model of the left ventricular wall. ${ }^{45,46}$ The model assumed an "ideal distribution" of fiber structure. From this, we incorporated the fiber disarray by altering the normal fiber orientation distribution through the addition of randomly generated offsets to the angles used to specify the myofiber orientation. The amount of randomness of the fiber orientation distribution was controlled by specifying the range over which random numbers were generated. Fiber disarray up to values of 4 deg exhibited no compromise in the systolic parameters. Disarray values of 10 deg or greater show ever-increasing degradation of systolic function, lower SVs, and lower EFs. The fiber strain results indicated that the loading on the fibers remains unchanged. The simulations suggested that the effects of disarray upon systolic function are pronounced whereas the diastolic function remains largely unchanged. Fusion of fiber-tracking results and the dispersion measures obtained in our present study from the same corresponding diffusion tensor data is a future project that could provide further insight into the spatial localization of specific pathology and its impact on heart function and may be useful in understanding the biomechanical ramifications of $\mathrm{LVH}$.

\section{Limitations}

This study is primarily limited by the small sample size and registration quality and accuracy arising from morphological differences between the WKY and SHR hearts and differences in sample handling. We noted an average Jaccard similarity coefficient of 0.70 with no statistically significant $(p=0.53$ ) difference between the coefficients for the WKY and SHR imaging data. Because of the small sample size we did not use a third group of WKYs to build the atlas, and then use two separate WKY and SHR groups to estimate dispersion from the atlas. The small sample size also did not show significant differences in heart/body ratios between the SHR and WKY populations, though in another one of our studies ${ }^{17}$ we saw significant differences. Additionally, the average time between fixation and imaging differed between the WKY and SHR populations. The effect of long-term storage in formalin on diffusion parameters and myocardial tissue microstructure is still not completely understood. It has been demonstrated that DTI parameters of myocardial tissue remain constant for a period of at least 7 days postfixation. ${ }^{47}$ However, a more recent study of the effects of long-term formalin fixation on myocardial tissue demonstrated statistically significant differences in FA and helix angle distribution. ${ }^{48}$ Previous studies of spinal cord tissue have found that postfixation diffusion anisotropy and diffusivity remain constant over time for a period of 15 weeks. ${ }^{49,50}$ Kim et al. ${ }^{50}$ suggest that storage in formalin does not cause a significant change in the direction of the principal eigenvector of the diffusion tensor. Despite these limitations, statistically significant differences were observed in the myocardial fiber dispersion measures between the wild-type and hypertrophic populations, which are consistent with the previous histological studies. $^{5,15}$

\section{Conclusion}

Our results demonstrate significantly altered myocardial fiber and laminar sheet structure in rats that suffer from hypertensive LVH. These results correlate with those obtained in human studies by Ferreira et al., ${ }^{14}$ which demonstrated abnormalities in laminar sheet orientation in hypertrophic myocardium. The dispersion data obtained can be combined with fiber-tracking methods to visualize myocardial changes in ventricular hypertrophy and understand the biomechanical ramifications of the remodeling process in the hypertrophic heart. ${ }^{6}$ The results make an important development for the strict quantification of changes in cardiac microstructure among various populations. Development of a normative myocardial microstructure as part of the analysis discussed in this study is expected to benefit other studies requiring group and longitudinal analyses.

\section{Acknowledgments}

The authors would like to thank Kathleen Brennan (LBNL) for harvesting the samples, and Osama Abdullah and Edward Hsu (University of Utah, Salt Lake City) for the diffusion tensor data collection. Additionally, Michael Miller and Hangyi Jiang at Johns Hopkins University provided valuable insight into the DiffeoMap software package. The authors would also like to thank Damien Rohmer (École Supérieure de Chimie Physique Életronique de Lyon) for assisting with the tensor analysis. This work was supported by the National Heart, Lung, and Blood Institute under Grant R01EB07219, and by the Director, Office of Science, Office of Biological and Environmental Research, Medical Sciences Division of the US Department of Energy under Contract DE-AC0205CH11231. 


\section{References}

1. M. C. Alvarez et al., "Is cardiac hypertrophy in spontaneously hypertensive rats the cause or the consequence of oxidative stress?," Hypertens. Res. 31(7), 1465-1476 (2008).

2. D. J. Breslin, R. W. Gifford, and J. F. Fairbairn, "Essential hypertension: a twenty-year follow-up study," Circulation 33, 87-97 (1966).

3. E. Kaplinsky, "Significance of left ventricular hypertrophy in cardiovascular morbidity and mortality," Cardiovasc. Drugs Ther. 8(Suppl. 3), 549-556 (1994).

4. B. H. Bulkley, M. L. Weisfeldt, and G. M. Hutchins, "Asymmetric septal hypertrophy and myocardial fiber disarray: features of normal, developing, and malformed hearts," Circulation 56(2), 292-298 (1977).

5. S. M. G. St. John et al., "Histopathological specificity of hypertrophic obstructive cardiomyopathy," Br. Heart J. 44, 433-443 (1980).

6. A. Giannakidis et al., "Diffusion tensor magnetic resonance imagingderived myocardial fiber disarray in hypertensive left ventricular hypertrophy: visualization, quantification, and the effect on mechanical function," in Cardiac Mapping, M. Shenasa et al., Eds., pp. 574-588, Blackwell Publishing Limited, Chichester, West Sussex, United Kingdom (2012).

7. E. W. Hsu et al., "Magnetic resonance myocardial fiber-orientation mapping with direct histological correlation," Am. J. Physiol. 274(5 Pt 2), H1627-H1634 (1998).

8. P. J. Basser, J. Mattiello, and D. LeBihan, "Estimation of the effective self-diffusion tensor from the NMR spin echo," J. Magn. Reson. 103(3), 247-254 (1994)

9. P. A. Helm et al., "Ex vivo 3D diffusion tensor imaging and quantification of cardiac laminar structure," Magn. Reson. Med. 54(4), 850-859 (2005).

10. J. M. Peyrat et al., "A computational framework for the statistical analysis of cardiac diffusion tensors: application to a small database of canine hearts," IEEE Trans. Med. Imaging 26(1), 1500-1514 (2007).

11. S. Sen et al., "Cardiac hypertrophy in spontaneously hypertensive rats," Circ. Res. 35, 775-781 (1974).

12. P. A. Helm et al., "Evidence of structural remodeling in the dyssynchronous failing heart," Circ. Res. 98(1), 125-132 (2006).

13. S. Angeli et al., "A high-resolution cardiovascular magnetic resonance diffusion tensor map from ex-vivo C57BL/6 murine hearts," J. Cardiovasc. Magn. Reson. 16, 77 (2014).

14. P. F. Ferreira et al., "In vivo cardiovascular magnetic resonance diffusion tensor imaging shows evidence of abnormal myocardial laminar orientations and mobility in hypertrophic cardiomyopathy," J. Cardiovasc. Magn. Reson. 16, 87 (2014).

15. I. J. Legrice et al., "Progression of myocardial remodeling and mechanical dysfunction in the spontaneously hypertensive rat," Am. J. Physiol. Heart Circ. Physiol. 303(11), H1353-H1365 (2012).

16. J. S. Huber et al., "Longitudinal evaluation of myocardial fatty acid and glucose metabolism in fasted and non-fasted spontaneously hypertensive rats using microPET/CT," J. Nucl. Med. 54(Suppl. 2), 390P (2013).

17. A. M. Hernandez et al., "Longitudinal evaluation of left ventricular substrate metabolism, perfusion, and dysfunction in the spontaneously hypertensive rat model of hypertrophy using small-animal PET/CT imaging," J. Nucl. Med. 54(11), 1938-1945 (2013).

18. M. D. Eggen, C. M. Swingen, and P. A. Iaizzo, "Ex vivo diffusion tensor MRI of human hearts: relative effects of specimen decomposition," Magn. Reson. Med. 67(6), 1703-1709 (2012).

19. S. H. Gilbert et al., "DT-MRI measurement of myolaminar structure: accuracy and sensitivity to time post-fixation, b-value and number of directions," in Conf. Proc. IEEE Engineering in Medical and Biology Society, pp. 699-702 (2013).

20. C. G. Koay et al., "A unifying theoretical and algorithmic framework for least squares methods of estimation in diffusion tensor imaging," J. Magn. Reson. 182, 115-125 (2006).

21. F. Beg et al., "Computing large deformation metric mappings via geodesic flows of diffeomorphisms," Int. J. Comput. Vision 61(2), 139-157 (2005).

22. G. Wu, Q. Wang, and D. Shen, "Registration of longitudinal image sequences with implicit template and spatial-temporal heuristics," NeuroImage 59(1), 404-421 (2012).

23. T. Vercauteren et al., "Diffeomorphic demons: efficient non-parametric image registration," NeuroImage 45(1), S61-S72 (2008).
24. P. Jaccard, "Nouvelles recherches sur la distribution florale," Bull. Soc. Vaudoise Sci. Nat. 44(163), 223-270 (1908).

25. D. Alexander et al., "An algorithm for preservation of orientation during non-rigid warps of diffusion tensor magnetic resonance (DT-MR) images," Proc. Int. Soc. Mag. Reson. Med. 9, 791 (2001).

26. A. Klein et al., "Evaluation of 14 nonlinear deformation algorithms applied to human brain MRI registration," NeuroImage 46(3), 786-802 (2009).

27. V. Arsigny et al., "Log-Euclidean metrics for fast and simple calculus on diffusion tensors," Magn. Reson. Med. 56(2), 411-421 (2006).

28. M. D. Cerqueira et al., "Standardized myocardial segmentation and nomenclature for tomographic imaging of the heart," Circulation 105(4), 539-542 (2002).

29. I. Kehat and J. D. Molkentin, "Molecular pathways underlying cardiac remodeling during pathophysiological stimulation," Circulation 122, 2727-2735 (2010).

30. M. Zhang, K. E. Sakaie, and S. E. Jones, "Logical foundations and fast implementation of probabilistic tractography," IEEE Trans. Med. Imaging 32(8), 1397-1410 (2013).

31. P.-T. Yap et al., "F-TIMER: fast tensor image morphing for elastic registration," IEEE Trans. Med. Imaging 29(5), 1192-1203 (2010).

32. W. Grossman, D. Jones, and L. P. McLaurin, "Wall stress and patterns of hypertrophy in the human left ventricle," J. Clin. Invest. 56, 56-64 (1975).

33. K. T. Weber et al., "Collagen remodeling of the pressure-overloaded, hypertrophied nonhuman primate myocardium," Circ. Res. 62, 757-765 (1988).

34. A. H. Gradman and F. Alfayoumi, "From left ventricular hypertrophy to congestive heart failure: management of hypertensive heart disease," Prog. Cardiovasc. Dis. 48, 326-341 (2006).

35. J. C. Rodrigues et al., "Comprehensive characterisation of hypertensive heart disease left ventricular phenotypes," Heart 102(20), 1671-1679 (2016).

36. O. M. Abdullah et al., "Characterization of diffuse fibrosis in the failing human heart via diffusion tensor imaging and quantitative histological validation," NMR Biomed. 27, 1378-1386 (2009).

37. J. C. Rodrigues et al., "ECG strain pattern in hypertension is associated with myocardial cellular expansion and diffuse interstitial fibrosis: a multi-parametric cardiac magnetic resonance study," Eur. Heart J. Cardiovasc. Imaging (2016).

38. L. Macron et al., "Global circumferential left ventricular strain impairment in hypertrophic cardiomyopathy: comparison to left ventricular hypertrophy and late gadolinium enhancement," J. Cardiovas. Magn. Reson. 15(Suppl. 1), E122 (2013).

39. G. Piellaa et al., "Characterizing myocardial deformation in patients with left ventricular hypertrophy of different etiologies using the strain distribution obtained by magnetic resonance imaging," Rev. Esp. Cardiol. 63, 1281-1291 (2010).

40. L. Iles et al., "Evaluation of diffuse myocardial fibrosis in heart failure with cardiac magnetic resonance contrast-enhanced T1 mapping," JACC 52, 1574-1580 (2008).

41. C. Y. Ho et al., "T1 measurements identify extracellular volume expansion in hypertrophic cardiomyopathy sarcomere mutation carriers with and without left ventricular hypertrophy," Circ. Cardiovasc. Imaging 6, 415-422 (2013).

42. V. Maestrini et al., "T1 mapping for characterization of intracellular and extracellular myocardial diseases in heart failure," Curr. Cardiovasc. Imaging Rep. 7, 9287 (2014).

43. E. Pozo et al., "Early gadolinium enhancement in hypertrophic cardiomyopathy: a potential premature marker of myocardial damage," Int. J. Cardiovasc. Imaging (2016).

44. D. Rohmer, A. Sitek, and G. T. Gullberg, "Reconstruction and visualization of fiber and laminar structure in the normal human heart from ex vivo diffusion tensor magnetic resonance imaging (DTMRI) data," Invest. Radiol. 42(11), 777-789 (2007).

45. A. I. Veress et al., "Incorporation of a left ventricle finite element model defining infarction into the XCAT imaging phantom," IEEE Trans. Med. Imaging 30, 915-927 (2011).

46. A. I. Veress et al., "Normal and pathological NCAT image and phantom data based on physiologically realistic left ventricle finite-element models," IEEE Trans. Med. Imaging 25, 1604-1616 (2006).

47. B. R. Watson and E. W. Hsu, "Effects of formalin fixation on diffusion tensor imaging of myocardial tissues" in Int. Society of Magnetic 
Resonance in Medicine 20th Annual Scientific Meeting, Melbourne, Australia (2012).

48. A. Giannakidis et al., "Value of formalin fixation for the prolonged preservation of myocardial microstructural organization: evidence by MR diffusion tensor imaging," Anat. Rec. 299(7), 878-887 (2016).

49. T. H. Kim et al., "Diffusion tensor imaging of ex vivo cervical spinal cord specimens: the immediate and long-term effects of fixation on diffusivity," Anat. Rec. 292(2), 234-241 (2009).

50. J. H. Kim et al., "Postmortem delay does not change regional diffusion anisotrophy characteristics in mouse spinal cord white matter," NMR Biomed. 20, 352-359 (2007).

Nicholas Tran received his master's of science degree in biomedical imaging at the University of California, San Francisco in 2015. $\mathrm{He}$ is a medical student at the University of Hawaii.
Archontis Giannakidis is an image analysis physicist with the Cardiovascular Biomedical Research Unit of Royal Brompton Hospital, London, UK. He also holds an honorary contract with the National Heart and Lung Institute at Imperial College London, London, UK.

Grant T. Gullberg is an adjunct professor of radiology at the University of California, San Francisco and is affiliated with the Lawrence Berkeley National Laboratory.

Youngho Seo is an associate professor of radiology, radiation oncology, and bioengineering at the University of California, San Francisco. $\mathrm{He}$ is also a group leader of the UCSF Physics Research Laboratory, Director of Nuclear Imaging Physics, and Director of the UCSF MicroPET/CT, MicroSPECT/CT, and Nuclear-Optical Imaging Core facility. 